\title{
Ultrastructure of vitreomacular traction syndrome associated with persistent hyaloid artery
}

A Gandorfer 1,2,3, M Rohleder ${ }^{1,2,3}$, D Charteris ${ }^{2}$, A Kampik ${ }^{1}$ and $\mathrm{P}$ Luthert $^{3}$

\section{Abstract}

Aim To demonstrate the ultrastructure of vitreomacular traction associated with persistent hyaloid artery.

Methods Pars plana vitrectomy was performed in a 66-year-old man with progressive vitreomacular traction associated with a persistent hyaloid artery. Epimacular tissue was peeled and processed for transmission electron microscopy. Results Ultrastructural analysis revealed multiple sheets of cellular and collagenous components. Myofibroblasts and newly formed collagen were the predominant features. Fibrous astrocytes, fibroblasts, macrophages, and basement membrane were also present.

Conclusion The cellular composition of the epimacular tissue and high cellular activity suggest that persistence of the hyaloid artery may contribute to the development of vitreomacular traction.

Eye (2005) 19, 333-336. doi:10.1038/sj.eye.6701459

Published online 16 July 2004

Keywords: persistent hyaloid artery; vitreomacular traction; epimacular pathology; macular surgery

\section{Introduction}

The embryonic hyaloid artery appears at the third week of gestation and reaches the lens by the fourth to fifth week to form the tunica vasculosa lentis. ${ }^{1,2}$ Atrophy of the hyaloid artery is usually complete before birth. ${ }^{3}$ Infrequently, however, anatomical remnants of the hyaloid system are found which may be manifest clinically as Mittendorf's dot or Bergmeister's papilla. ${ }^{4}$ Persistence of the hyaloid artery with active blood flow is a rare finding. ${ }^{1}$ There are several reports of vitreous haemorrhage and traction retinal detachment in association with persistence of the hyaloid artery. ${ }^{5-9}$ Out of a total of almost 20 patients with persistent hyaloid artery reported in the literature, only two cases showed vitreoretinal traction. ${ }^{7,10}$ In one patient, there was traction retinal detachment next to the optic nerve head involving the macula. ${ }^{7}$ The other patient had traction maculopathy without detachment of the retina. ${ }^{10}$

This study reports on the ultrastructure of epimacular tissue in a patient with vitreomacular traction syndrome (VMTS) in association with a previously diagnosed persistent hyaloid artery.

\section{Patient and methods}

A 66-year-old man complained of progressive visual decline in the right eye for 18 months.

Visual acuity was 6/120 in the right eye, and 6/ 6 in the left eye. On slit-lamp examination, there was marked cataract formation in the right eye with otherwise unremarkable anterior segments in both eyes. Biomicroscopy of the posterior pole of the right eye revealed a persistent hyaloid artery, which had been diagnosed some years before, and mild diffuse vitreous haemorrhage. The vitreous was incompletely detached from the retina with clinically obvious persisting vitreous strands to the optic nerve head and to the macula. There was epiretinal membrane formation over the posterior pole with surface wrinkling of the macula and distortion of retinal vessels. The posterior segment of the fellow eye was unremarkable.

Combined pars plana vitrectomy with phacoemulsification and lens implantation were
${ }^{1}$ Department of Ophthalmology

University Eye Hospital Ludwig-MaximiliansUniversity

Munich, Germany

${ }^{2}$ Moorfields Eye Hospital Vitreoretinal Research Unit London EC1V 2PD, UK

${ }^{3}$ Department of Pathology Institute of Ophthalmology London EC1V 9EL, UK

Correspondence:

A Gandorfer

Department of

Ophthalmology

University Eye Hospital

Ludwig-Maximilians-

University Mathildenstr. 8

Munich 80336

Germany

Tel: +498951603800

Fax: + 498951604778

E-mail: arnd.gandorfer@

ak-i.med.uni-muenchen.de

Received: 20 August 2003

Accepted: 12 January 2004 Published online: 16 July 2004 
performed. Posterior vitreous detachment was initiated with the vitrectomy probe over the posterior pole, and continued peripherally. The vitreous was firmly attached to the optic nerve head and to the macular area. The vitreous strands to the optic disc were cut with the vitrectomy probe. Diathermy of the persistent hyaloid artery was performed at a distance of approximately $500 \mu \mathrm{m}$ from the optic disc to avoid damage to other

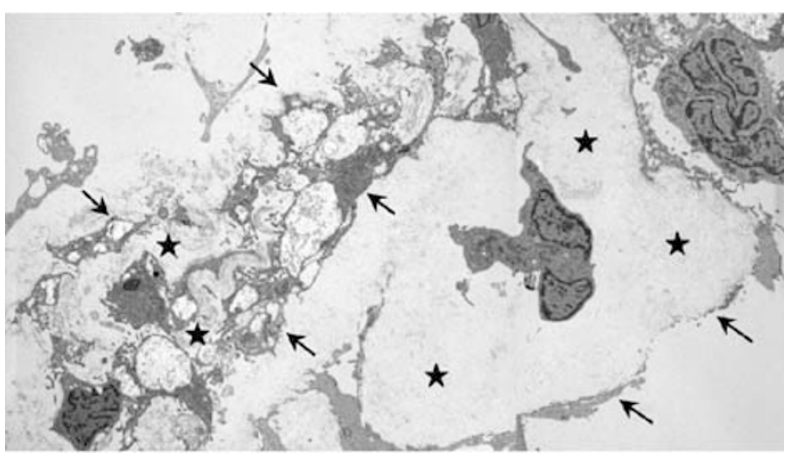

Figure 1 Epimacular membrane associated with persistent hyaloid artery. Arrows indicate cellular layers, stars indicate fibrous component interposed between the cellular layers. $\times 1800$. retinal vessels. The epiretinal membrane was peeled from the macula using an end-gripping forceps. Postoperatively, visual acuity improved to $20 / 25$, without recurrence of epimacular membrane.

The specimen was fixed in phosphate-buffered $4 \%$ glutaraldehyde. The tissue was postfixed with Dalton's fixative (osmium 2\%), dehydrated and embedded in Epon. Semithin sections were stained with $2 \%$ toluidine blue for light microscopy. For electron microscopy, ultrathin sections were contrasted with uranyl acetate and lead citrate. Tissue sections were analyzed using a Zeiss EM 9 S-2 electron microscope.

\section{Results}

Ultrastructural examination of epimacular tissue revealed a multilayered structure consisting of a cellular and a fibrous component (Figure 1). Myofibroblasts were the predominant cell type, and were characterised by a lobulated nucleus and aggregates of 5-7-nm subplasmalemmal cytoplasmatic filaments with fusiform densities (Figure 2a and b). Fibrous astrocytes, fibroblasts, and macrophages were also present, and showed ultrastructural features of high metabolic activity
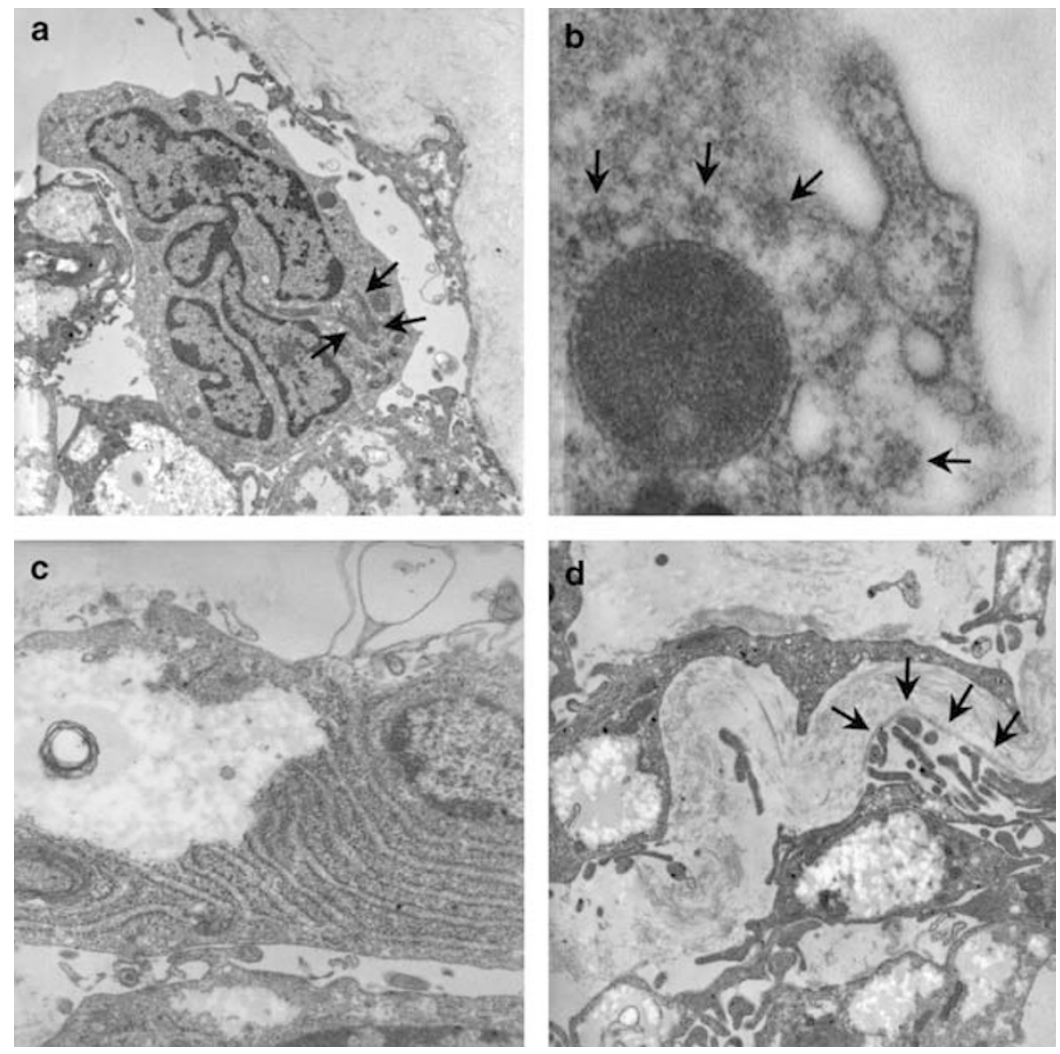

Figure 2 (a): Myofibroblast with lobulated nucleus and mitochondria (arrows). $\times$ 4800. (b): High magnification of 2(a). Aggregates of intracytoplasmatic microfilaments (arrows) characteristic of myofibroblasts. $\times 60000$. (c): Fibroblasts with masses of rough endoplasmatic reticulum. $\times 15000$. (d): Basal membrane (arrows) interspersed between cells and collagen. $\times 9500$. 
such as prominent mitochondria and masses of rough endoplasmatic reticulum (Figure 2c). Retinal pigment epithelial cells were absent. The cells were arranged in multiple sheets with the fibrous component interposed between the cellular layers. The fibrous component consisted mostly of newly formed collagen characterised by a collagen fibril with diameter of more than $16 \mathrm{~nm}$. Areas of basement membrane formation were also present (Figure 2d). Internal limiting membrane was not found in any of the specimens.

\section{Discussion}

Previous work on the ultrastructure of VMTS has shown two distinct types of epiretinal membranes: (a) single cells or a cellular monolayer in direct contact with the vitreal side of the internal limiting membrane, and (b) multilayered fibrocellular tissue separated from the internal limiting membrane by native collagen. ${ }^{11}$ Clinically, only the latter type can be identified as a clearly visible epimacular membrane. From the clinical and histopathological point of view, one might expect the present case to be analogous to a type (b) membrane configuration. Ultrastructure, however, reveals clear differences between this case and the previously reported specimens of VMTS. Patients with VMTS and associated epimacular membranes consistently had one densely packed cellular component and a single continuous layer of native vitreous collagen interposed between the cellular layer and the internal limiting membrane. In contrast, the present case had multiple layers of cellular proliferation, with newly formed collagen interspersed in between. The multilayered cellular components tended to be arranged more loosely. Notably, macrophages were frequently found in the present case but were not observed in previously examined specimens of VMTS. Basement membrane formation was seen in the present case. Overall, the morphology of this specimen suggested an active state of cellular remodelling despite a history of 18 months duration of visual decline: there was plentiful rough endoplasmatic reticulum and abundant mitochondria were present, and large amounts of new collagen had formed. The preponderance of myofibroblasts and the occurrence of macrophages with multiple intracytoplasmic vesicles is additional evidence of continued metabolic activity.

The present case also shared common features with previous descriptions of specimens of VMTS. Notably, myofibroblasts were the predominant cell type. Ultrastructural features of cellular activity have been seen in previous specimens of VMTS, although these features were much more pronounced in our case. Myofibroblasts, fibroblasts, and fibrous astrocytes were present both in this case and in the previous VMTS cases studied.

Incomplete regression of the embryonic vitreous with persistence of the hyaloid artery implies attachment of the vitreous to the optic disc which may result in vitreoretinal traction. ${ }^{9,10}$ However, it does not usually result in either vitreous strands or traction to the macula nor in the development of an epimacular membrane.

Vitrectomy has been shown to release vitreomacular traction effectively. However, diathermy of a persistent hyaloid artery may be complicated by the occlusion of a branch retinal artery. ${ }^{7}$ As recommended previously, care should be taken to consider atypical branches of retinal arteries which may be affected by diathermy. ${ }^{7}$ Regarding the distance between the coagulation site and the retina, there is no data in the literature which allows to determine a safe coagulation distance without damaging essential structures. In the present case, there were no complications of diathermy. Whether this was due to a sufficient distance between the coagulation site and the retina, or due to the lack of atypical branches of retinal arteries cannot be concluded from this case.

Ultrastructural analysis of a single case, at one timepoint cannot finally elucidate the relationship of a persistent hyaloid artery with the subsequent development of VMTS. Persistence of the hyaloid artery could, however, prevent complete posterior hyaloid separation or contribute to epiretinal membrane formation, or possibly both. Notably, both VMTS and persistent hyaloid artery are characterised by abnormally firm attachment of the vitreous to the posterior pole as evidenced by reports of vitreomacular traction and tractional retinal detachment in eyes with persistence of the hyaloid artery. ${ }^{7,10}$ In comparison to previous reports of idiopathic VMTS, the greater cellular activity, larger population of cells, and marked collagen deposition of the present case are evidence that the persistence of the hyaloid artery can provide or enhance vitreomacular traction by promoting epiretinal membrane development.

\section{References}

1 Brown G, Tasman W. Congenital Anomalities of the Optic Disc. Grune and Stratton, New York, 1983, pp 58-71.

2 Mann IC. Development of the Human Eye. Grune and Stratton, New York, 1964; 210-214.

3 Jack RL. Regression of the hyaloid vascular system: an ultrastructural analysis. Am J Ophthalmol 1972; 74: 261-272.

4 Zimmermann LE, Font RL. Congenital malformations of the eye. JAMA 1966; 196: 684.

5 Delaney WV. Peripapillary hemorrhage and persistent hyaloid artery. Am J Ophthalmol 1980; 90: 419-421. 
6 Unoki K, Nakao K, Ohba N. Hemorrhage in the lens: spontaneous occurrence in congenital cataract. $\mathrm{Br} \mathrm{J}$ Ophthalmol 1986; 70: 593-595.

7 Thumann G, Bartz-Schmidt KU, Kirchhof B, Heimann K. Branch retinal artery occlusion by diathermy of a persistent hyaloid artery. Am J Ophthalmol 1997; 124: 415-416.

8 Manschott W. Persistent hyperplastic primary vitreous. Arch Ophthalmol 1958; 59: 188-203.
9 Pruett RC. The pleomorphism and complications of posterior hyperplastic primary vitreous. Am J Ophthalmol 1975; 80: 625 .

10 Cockburn DM, Dwyer PS. Posterior persistent hyperplastic primary vitreous. Am J Optom Physiol Opt 1988; 65: 316-317.

11 Gandorfer A, Rohleder M, Kampik A. Epiretinal pathology of vitreomacular traction syndrome. Br J Ophthalmol 2002; 86: 902-909. 